\title{
Animal experiments for the determination of an optimal wavelength for retinal coagulations
}

\author{
Martin Vogel $^{1}$, Fritz Peter Schäfer ${ }^{2}$, Michael Stuke ${ }^{2}$, Kurt Müller $^{2}$, Sabine Theuring ${ }^{1}$, and Alina Morawietz ${ }^{1}$ \\ ${ }^{1}$ Universitäts-Augenklinik, Robert-Koch-Strasse 40, D-3400 Göttingen, Federal Republic of Germany \\ ${ }^{2}$ Abteilung Laserphysik, Max Planck Institut für biophysikalische Chemie, Am Fassberg, D-3400 Göttingen, \\ Federal Republic of Germany
}

\begin{abstract}
The retina of rabbits was coagulated with different wave-lengths $(570-630 \mathrm{~nm})$ using a tunable dye laser with Rhodamin 6G. To achieve comparable ophthalmoscopic appearance the intensity of the laser beam was varied with neutral filters of varying absorption. Histologic examination of fresh coagulation effects and 3-week-old coagulation scars showed no difference in relation to the wavelength used. Theoretically, $577 \mathrm{~nm}$ seems to be a particularly useful wavelength for the treatment of a wide variety of retinal lesions because it is not absorbed in xanthophyll and penetrates opacities of the optic media better than argon blue green. Furthermore, it is absorbed maximally in hemoglobin, making it particularly suitable for the coagulation of blood vessels.
\end{abstract}

\section{Introduction}

The dye laser with the capacity of emitting different wavelengths was first introduced into ophthalmology in 1984 [3]. On the basis of the discussions that evolved from a number of publications $[1,2,5-7,9,10]$ over the advantages and disadvantages of the argon and krypton laser, it appeared desirable to have a laser that emits the wavelengths of the spectrum continuously. Such lasers are available. Usually an argon laser tube pumps two or three dye modules. This means a high price for a large complex instrument. Using an argon laser-pumped dye laser with Rhodamin $6 \mathrm{G}$ we examined whether different wavelengths really have advantages in clinical retinal coagulations and which wavelength would lend itself best for clinical use. The wavelengths $570-630 \mathrm{~nm}$ were at our disposal in a continuous range. They were used for the coagulations of rabbit retinas.

\section{Material and methods}

In six anesthesized Chinchilla rabbits, of the same breed and with an evenly pigmented fundus, laser coagulation effects were placed underneath the myelinated nerve fibers. The spot size was $500 \mu \mathrm{m}$. The wavelengths $620,604,592$, and $577 \mathrm{~nm}$ were used. The goal of the coagulations was to produce lesions ophthalmoscopically as identical as possible. To accomplish this, neutral filters of varying absorption were used to modify the intensity of the effects. This

Offprint requests to: $\mathrm{M}$. Vogel was particularly important when coagulating blood vessels because the range from no effect to rupture of the vessel was narrow.

In one rabbit the eyes were removed $48 \mathrm{~h}$ after laser treatment. They were opened with an Elliot's trephine, fixed in cold 4\% glutaraldehyde solution, and embedded in paraffin for light microscopic examination. The sections were stained with hematoxylin and eosin. The eyes of the other animals were removed 3 weeks after laser coagulation and prepared accordingly.

\section{Results}

With all used wavelengths, ophthalmoscopically identical effects of varying intensity could be produced on the fundus of the rabbit (Fig. 1).

The histologic examination of the eyes removed $48 \mathrm{~h}$ after laser coagulation revealed edema and partial destruction of the sensory retina. The changes reached up into the nerve fiber layer. The retinal pigment epithelium (RPE) was partially necrotic (Fig. 2). In no instance was there disruption of Bruch's membrane or choroidal hemorrhage.

In those eyes removed 3 weeks after laser coagulation scar formation was completed. There were no signs of exudation or inflammatory cells. There was marked proliferation of the RPE into the sensory retina. The architecture of the retina had disappeared in favor of glial scar (Fig. 3). The histologic slides received code numbers and were distributed to two independent examiners who did not know which wavelengths were used. They could not observe any differences between the retinal scars in relation to the applied wavelength.

\section{Discussion}

The effect of different wavelengths on the fundus has been the subject of several studies $[1,2,5-9,11,12]$ concerning krypton red and argon green. The investigators agree that krypton red, because of its lower absorption in the RPE, extends into the choroid and may cause hemorrhage in this tissue. Coagulation with krypton red may be more painful and, therefore, may require local anesthesia $[1,4,8,10]$. Absorption of krypton red is low in hemoglobin and lends itself well in the case of vitreous hemorrhage, provided one can see the fundus. Using krypton red requires care. It should only be applied with barely visible effects because grayish white effects, as desired with argon green, would 

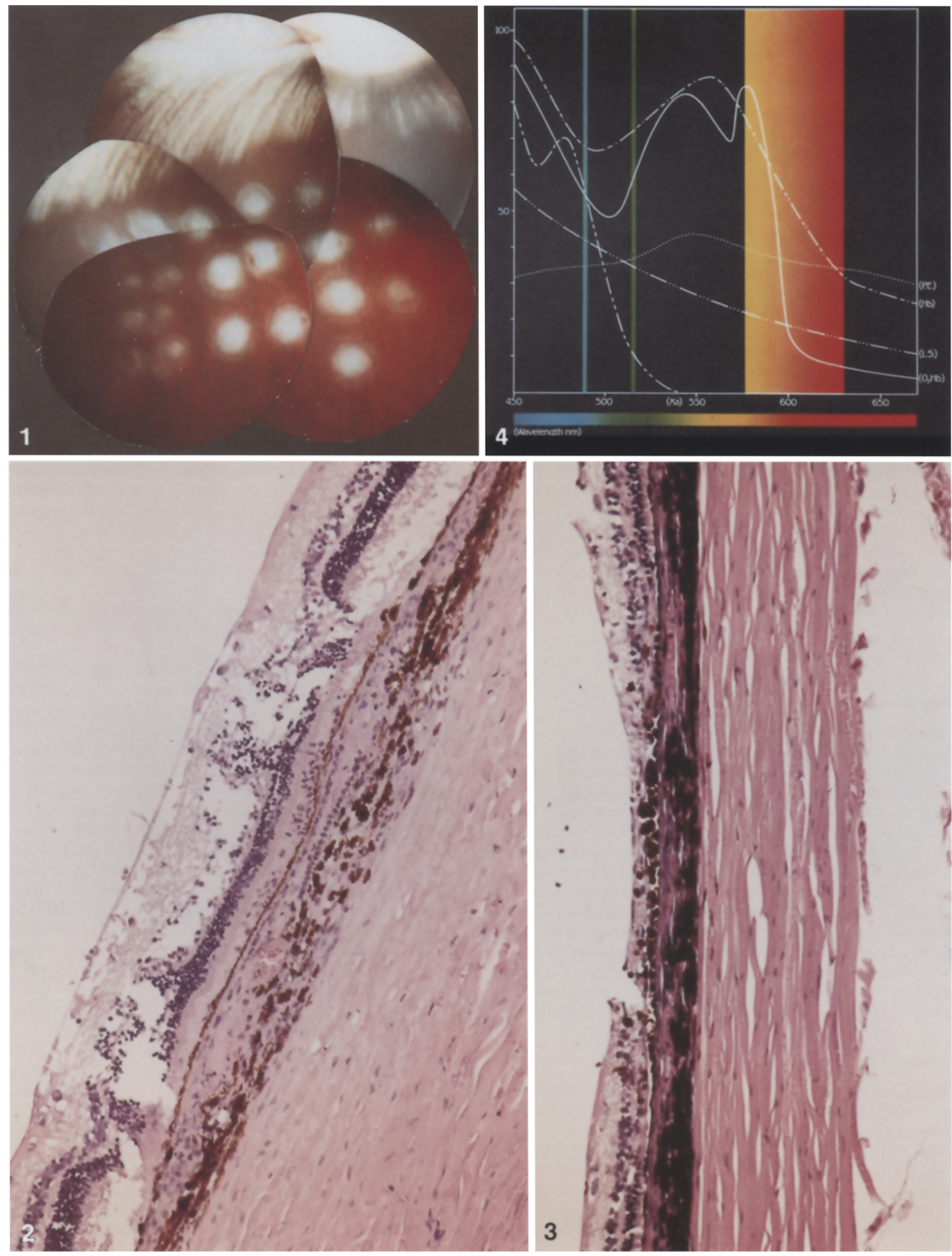
be much too strong and would lead to choroidal hemorrhage. It is currently still under investigation whether this wavelength is advantageous in routine coagulations of subretinal macular vascular proliferations or in retinal vein occlusion.

The blue-green argon laser has the disadvantage that its light is partially absorbed by the macular xanthophyll. This, however, can only take place in the normal macula because in those cases where there is edema in the macular area the xanthophyll is diluted and, in view of its low concentration, the amount of light absorbed is minimal.

With the dye laser we are able to use other wavelengths besides krypton red and argon green. On the basis of the absorption curves of the optic media, xanthophyll, RPE, as well as oxygenated and deoxygenated hemoglobin, several authors $[2,3,8,10]$ have demonstrated which wavelength would be most effective. For penetration of vitreous hemorrhage $650 \mathrm{~nm}$ would be ideal and for effects in the RPE $550 \mathrm{~nm}$. At between 500 and $700 \mathrm{~nm}$ there will be hardly any absoprtion in macular xanthophyll. The dye laser offers these wavelengths (Fig. 4). One must, however, realize that these calculated wavelengths are only significant if one works with barely visible threshold lesions. As a rule we need lesions far above threshold for therapeutic purposes. Since by far the highest absorption for all wavelengths lies in the RPE, above threshold lesions will lead to comparable reactions in the RPE, no matter which wavelength is used. This means that in clinical use the advantages or disadvantages of different wavelengths are neutralized. Only krypton red, because of its extremely long wavelength, penetrates the RPE more readily. Whether this is desirable is still a matter of dispute.

Our histological examinations confirm the ophthalmoscopic observations. We have placed comparable acute effects into the rabbit retina using a variety of wavelengths, yet, in the stage of early healing one can not see any difference. Therefore, equatorial degenerations and other nonvascular lesions of the retina probably can be treated successfully with any wavelength as clinical experience suggests.

For the treatment of vascular lesions and subretinal senile macular proliferations the ideal would be a wavelength which is maximally absorbed in hemoglobin. One of the absorption maxima of hemoglobin lies at $577 \mathrm{~nm}$. This wavelength is located at the short wavelength end of the emis- sion curve of Rhodamin 6G. High energy is necessary to produce adequate $577 \mathrm{~nm}$ light for therapeutic purposes; however, it has the following ideal characteristics:

1. It is not absorbed in xanthophyll.

2. It penetrates opacities of the media with $30 \%$ less energy than argon blue-green because longer wavelengths are dispersed less than short wavelengths. Opaque media, therefore, suffer less heating.

3 . It is not located far from the absorption maximum of the RPE.

4. Because of the high absorption in hemoglobin, obliteration of small vessels requires less energy than the coagulation with argon blue-green. A slow approach to the therapeutic level is recommended because larger vessels may rupture, probably as a result of the high absorption in hemoglobin.

According to the theoretical considerations and the experimental results, a laser emitting $577 \mathrm{~nm}$ will serve all purposes. We have developed a flashbulb-pumped dye laser emitting $577 \mathrm{~nm}$ alone. It has stood the test in animal experiments, we will report on this compact instrument in the near future.

\section{References}

1. Apple DJ, Thomas G, Swartz M, Kavka-van Norman D (1984) Comparative histopathology and ultrastructure of human krypton and argon lesions. Doc Ophthalmol Proc Ser 36:45-60

2. L'Esperance FA (1968) The ocular histopathologic effect of krypton and argon laser radiation. Am $\mathrm{J}$ Ophthalmol 68:263-273

3. L'Esperance FA (1984) Clinical applications of the organic dye laser. Ophthalmology 92:1592-1600

4. L'Esperance FA (1985) Trans-spectral organic dye laser photocoagulation. Trans Am Ophthalmol Soc 83:82-113

5. Gabel V-P, Birngruber R, Lachemayr B (1984) Ist der KryptonLaser anderen Lichtquellen zur Koagulation der Netzhaut überlegen? Fortschr Ophthalmol 81:150-153

6. Marshall J, Bird AC (1979) A comparative histopathological study of argon and krypton laser irradiations of the human retina. Br J Ophthalmol 63:657-668

7. Marshall J, Clover G, Rothery S (1984) Some new findings of retinal irradiation by krypton and argon lasers. Doc Ophthalmol Proc Ser 36:21-37

8. Peyman GA, Raichand M, Zeimer RC (1984) Ocular effects of various laser wavelengths. Surv Ophthalmol 28:391-404

9. Sabates FN, Lee KY, Ziemianski MC (1982) A comparative

Fig. 1. Photograph of the rabbit fundus. By using neutral filters of different degrees of absorption, coagulation effects of varying intensity from very mild to strong with small hemorrhages were produced; these were $500 \mu \mathrm{m}$ effects. Such lesions could be coagulated with all wavelengths

Fig. 2. Cross section of the rabbit retina $48 \mathrm{~h}$ after laser coagulation with $560 \mathrm{~nm}(\mathrm{HE}, \times 80)$. The heat ball developing in the retinal pigment epithelium involves the rods and cones and the outer nuclear layer completely. The inner layers of the retina show disintegration, which is also partly caused by artifacts. The wavelengths used in these experiments lead to identical lesions. The histologic examiner, was unable to determine which wavelength was used

Fig. 3. Cross section of the rabbit retina 3 weeks after laser coagulation $(610 \mathrm{~nm}, \mathrm{HE}, \times 60)$. The retinal tissue is replaced by glia and proliferation of the retinal pigment epithelium. In the scar formation, as well, no identification of the used wavelength could be made

Fig. 4. Absorption diagram of xanthophyll, retinal pigment epithelium, oxygenated and deoxygnated hemoglobin, and the emission spectrum of Rhodamin 6G. At the short wave end of Rhodamin $6 \mathrm{G}$ is $577 \mathrm{~nm}$ which coincides with the maximum of doxygenated hemoglobin and lies close to the absorption maximum of the retinal pigment epithelium and oxygenated hemoglobin; 577 nm is not absorbed in xanthophyll. The green and blue-green columns indicate the emission of argon lasers (courtesy of Coherent Radiation) 
study of argon and krypton laser photocoagulation in the treatment of presumed ocular histoplasmosis syndrome. Ophthalmology 89:729-734

10. Schulenburg WE, Hamilton AM, Blach RK (1979) A comparative study of argon laser and krypton laser in the treatment of diabetic optic disc neovascularisation. $\mathrm{Br} \mathrm{J}$ Ophthalmol 63:412 417

11. Wieder M, Pomerantzeff O, Schneider J (1981) Retinal vessel photocoagulation: a quantitative comparison of argon and krypton laser. Invest Ophthalmol 20:418-424

12. Wollensak J, Seiler R (1985) Der Berliner Farbstofflaser.

Fortschr Ophthalmol 82:606-608

Received May 26, 1988/Accepted September 14, 1988 